\title{
Monocytes to lymphocytes ratio in peripheral blood and immunoglobulin IgE levels as indicators to Plasmodium falciparum infection in Sudan
}

\begin{abstract}
Background: Malaria infection especially by Plasmodium falciparum species remain the most global threating life in Africa. The effective immune response to malaria infection, in addition to the effective immunological factors which play a significant role in immune defence reflect in different sorts of infection, such as changes in cellular ratio and the elements quantity according to the immunological challenge still are incompletely understood. In this survey, we examined the ratio of monocytes to lymphocytes beside the levels of total immunoglobulin IgE in both infected patients with Plasmodium falciparum parasite and compared with healthy individual. To search for any correlation on those who had the infection.
\end{abstract}

Methodology: We obtained $3 \mathrm{ml}$ of venous blood samples from both infected patients and control candidates in EDTA containers for immunoglobulin inspection and parasitological tests. A full differential blood count, Immuno-chromatography antigenic test and thin blood films with a microscopic examination had been used to confirm malaria infection. In addition to Electro-chemiluminescence-immunoassay (ECL) had been applied for measuring the total immunoglobulin IgE levels. We entered Data into Microsoft word and analyzed using SPSS (version 20; SPSS Inc., Chicago, IL) software, the correlation to occurrences of malaria infection and between variables calculated using Pearson correlation coefficient. An alpha value of $<0.05$ denoted a statistically significant difference in all statistical compares.

Finding: The analysis of the results revealed that the IgE levels were dependent with the incidence of the malaria infection P-value $<0.01$. Furthermore, the ratio of Monocytes to Lymphocytes (M: $\mathrm{L})$ elevated with the risk of malaria infection $(\mathrm{HR}=0.30,95 \% \mathrm{CI}=0.11$ to 0.84 ) and found to be statistically significant $\mathrm{P}$-value $<0.05$.

Conclusion: The outcome of this work revels that, both the monocytes to lymphocytes (M: L) ratio and the $\operatorname{IgE}$ could be used for predication of the malaria infection as well as can used as a good indicator to monitor the density of malaria infection.

Keywords: malaria infection, Plasmodium falciparum, monocytes to lymphocytes ratio (m: 1 ratio), total immunoglobulin $\mathrm{E}$
Volume 7 Issue 6 - 2019

\author{
Dia Aldeen Alfaki,' Ibrahim Mohamed Eisa, ${ }^{2}$ \\ Mohamed Mubarak Elbasheir, ${ }^{2}$ Mohammed \\ Elfatih Humida ${ }^{3}$ \\ 'Department of Hematology, Alzaeim al azhari university, Sudan \\ ${ }^{2}$ Department of Parasitology, Alzaeim al azhari university, Sudan \\ ${ }^{3}$ Department of Microbiology, Orotta College of Medicine and \\ Health Sciences, Eritrea
}

\begin{abstract}
Correspondence: Dia Aldeen Alfaki, Faculty of Medical Laboratory Alzaeim al azhari university, Khartoum Sudan, Tel +971554040895, Email alfaki-di@outlook.com
\end{abstract}

Received: November 05, 2019 | Published: November 29 2019

\section{Introduction}

Plasmodium falciparum malaria is still a major cause of morbidity and mortality in Africa, where the greatest burden of disease is borne by young children; Substantial clinical immunity develops following repeated natural exposure to Plasmodium falciparum such that clinical malaria tends to be less frequent in children over 5years of age and adults. This distinction between immunity to clinical malaria and immunity against Plasmodium falciparum infection per she is further evident in the epidemiological pattern of clinical malaria and asymptomatic parasitemia. The specific host factors underlying susceptibility to clinical malaria despite the ability to sustain asymptomatic Plasmodium falciparum infection is poorly understood. Worldwide, most infections with malaria-causing agents are clinically silent, reflecting the ability of adaptive immune mechanisms to prevent disease. ${ }^{2}$ Patients infected with malaria exhibited important changes in most of hematological parameters, including low platelets, white blood cells (WBCs) and lymphocytes counts being the most important predictors of malaria infection. When use these parameters in combination with other clinical and microscopy methods could improve the malaria diagnosis and treatment. Hematological changes are the most common indicators of malaria infection as well as play a significant role in pathology of malaria. These changes involve the major cells types such as RBCs, leucocytes and thrombocytes. ${ }^{3}$

Furthermore, the main role of white blood cells (WBCs) counts and their differential types are basic and essential indicators resulting from infection. Which serve and help to differentiate between different types of infections and serve as a tool in monitoring the patient's progress during illness, ${ }^{4}$ which given the central role of monocytes and lymphocytes in the induction of immune responses, moreover, their frequency in peripheral blood might be expected to reflect the state of an individual's immune-response to infection. ${ }^{1,5}$ Mechanisms for malaria immunity are highly complex with involvement of several components of immune function, Antibody-dependent mechanisms are presumed to play an important role in protection, with a wide range of antigen specific antibodies as well as polyclonal-antibody production. ${ }^{6}$ Structurally different antigens are expressed during each part of the parasite's life cycle, hence naturally acquired immunity is mostly stage specific. Both antibodies and $\mathrm{T}$ cells are required for naturally acquired immunity. Antibodies may block host cell invasion by sporozoites and merozoites, ${ }^{6} \mathrm{IgE}$ interaction with effectors cells is assumed to play an important although controversial role in protection against parasitic infections. ${ }^{7}$ In Plasmodium falciparum infection 
induces elevated blood levels of both total immunoglobulin and antiplasmodium antibodies belonging to different isotypes. It has been previously shown that donors living in areas of malaria transmission develop malaria-specific IgE antibodies that are present at highest concentrations in patients with severe disease, suggesting a role for this isotype in malaria pathogenesis. ${ }^{5,8}$

\section{Materials and methods}

\section{Study design}

A cross sectional study was conducted at Dar AL-elaj Specialized Hospital (D.S.H), Khartoum state, Sudan from March to July 2017.

\section{Study population}

Sudanese patients, infected by $P$. falciparum malaria, the population categorized according to age group (adult and children less than 14 a years) and gender. The patients with previous history of malaria infection within last 3 months or how suffering from immunological disorders were excluded from study.

\section{Sampling and preparation}

After obtaining the permission and inform consent from the treating doctors and each volunteers or patients informed with the aim of the study. A total of 126 were enrolled during the study period and $3 \mathrm{~mL}$ venous blood collected from all participants. 76 samples from admitted patients in (DSH) hospital with Plasmodium falciparum malaria infection and 50 samples from healthy individual's volunteer as controls. All laboratory tests were performed within 2 hours from the time of collection.

\section{Parasitological analysis}

The immuno-chromatography assay was used for the first detection of HRP-II of Plasmodium falciparum malaria. thin blood films were prepared on clean frosted slide, dry, grease free and labeled and stained using Leishman stain for confirming and determining the percentage counting of parasitic density Using the formula in count: Number of infected RBCs Per 1000 RBCs cells * 100 .

\section{Full WBCs differential count}

WBCs count and differential performed by using the full automated hematological analyzer system. XT-1800i., and the M: $\mathrm{L}$ ratio calculated for each participant. Thin blood film also used to confirm the ratio and the cell morphology.

\section{Immunoglobulin IgE estimations}

All Samples drawn in (EDTA) were centrifuged for 2500 Rounds per 5 minutes and then separated the plasma in COBAS samples cup $0.5 \mathrm{ml}$. All samples were tested for total Immunoglobulin (IgE) assay measurement using full automated COBAS e411 and IgE levels were reported in international units.

\section{Statistical analysis}

Statistical analysis was conducted in this study using SPSS (version 20; SPSS Inc., Chicago, IL) software. Data were expressed as mean \pm standard deviation. Comparisons of continuous variables made using the Student's t-test for parametric data. The correlations between Immunoglobulin E Levels, TWBC and Leucocyte differential variables calculated using Pearson correlation coefficient. An alpha value of $<0.05$ denoted a statistically significant difference in all statistical compares.

\section{Result}

A total of 126 from all study groups analyzed for total IgE. In stark contrast, however infected patients have the highest level of total $\mathrm{IgE}$ (ranging from 28 to $2105 \mathrm{IU} / \mathrm{ml}$ ) and this difference highly statistically significant $(\mathrm{P}$-value $<0.001)$ furthermore $\mathrm{M}$ : $\mathrm{L}$ ratio $(0.49)$ found to be a highly statistically significant difference too (P-value $<0.001$ ) (Table 1). In patient with severe malaria the total IgE level meanwhile, varied from $392.56 \mathrm{IU} / \mathrm{ml}$ (under 15years) to $369.93 \mathrm{IU} / \mathrm{ml}$ (more than 45years) and this group have a higher level than the other group 15-29 \& 30-44 years old (209.12 \& 161.25 respectively). All these differences were significant $(\mathrm{P}-$-value $<0.05)$ Table 2. Reporting the correlation between the white blood cell count and differential have negative correlation in spite of monocytes significantly positive with severity of malaria $(\mathrm{P}$-value $<0.05)$ and the correlation of immunoglobulin levels with severity of malaria illustrated in (Table 3). Total $\operatorname{IgE}$ have a positive correlation with severity of malaria (P-value $<0.001) . \mathrm{M} / \mathrm{L}$ ratio associated with the risk of clinical malaria ( $\mathrm{HR}=0.30,95 \% \mathrm{CI}=0.11$ to 0.84 ) found to be statistically significant $\mathrm{p}$-value $<0.05$. The analysis of the results revealed that the IgE levels Table 4. The relative frequency of monocytes to lymphocytes in peripheral circulation reflects on the infected individuals'. Show the considerable an increase in an $\mathrm{M} / \mathrm{L}$ ratio with the density of the infection by Plasmodium falciparum (Figure 1). The irregular Climb in the level of immunoglobulin with the rate of the infection by Plasmodium falciparum reported in patient groups (Figure 2). Total IgE levels have been high already in the youngest age group $(<15$ years), also appeared to be higher in older age patients ( $>45$ years). The apparent decrease in concentrations between 15-44years old and this difference was statistically significant Figure 3.

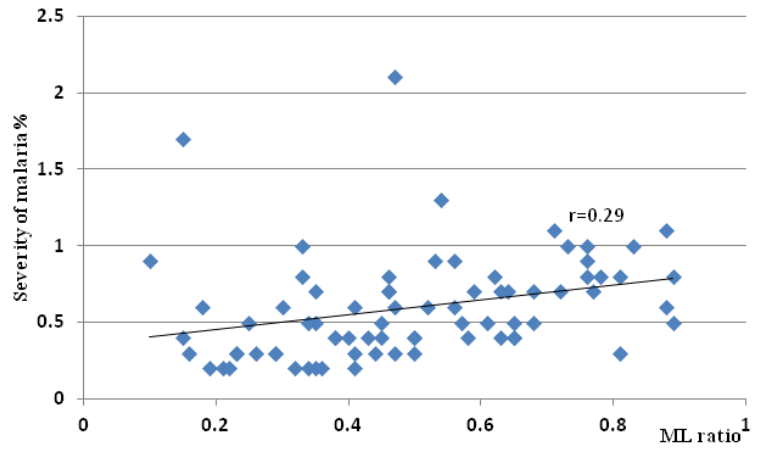

Figure I Correlation between $\mathrm{M} / \mathrm{L}$ ratio and severity of malaria.

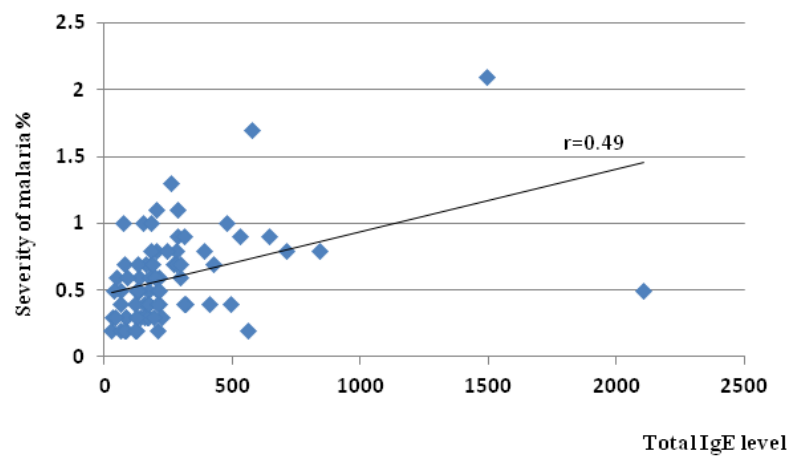

Figure 2 Correlation between total lgE levels and severity of malaria. 




Figure 3 Mean of total lgE level in different age groups in malaria patients.

\section{Discussion}

The study purpose was to find whether if there is any significant correlation, between the specific cellular compounds of the immune system which measured. And total IgE with the infection of malaria by Plasmodium falciparum species. Results revealed that there is slight reduction in total white blood count during the infection when compared against the control group, which is in line with what has been found in antecedent studies. ${ }^{4,9}$ This droop in total might due to the exhaustion in the immunological process to fight out the parasitic invasion. We found that there is light change in WBCS parameter (neutrophil, lymphocytes, monocytes and eosinophils according to each formed with his particular role. To expel the pathogenicity of Plasmodium falciparum, which adapt by wide range of interleukin's. ${ }^{10}$ It was statistically different on the infected patient than controls group (Table 3), and this finding Homogeneously are aligned with similar articles. ${ }^{4,11} \mathrm{M}$ : L ratio was found to be highly statistically significant different, when it compared with the control group (Table 1).

Table I Distribution of the total immunoglobulin E (IgE) levels and monocyte to lymphocyte ratio (ML ratio) in the studied populations

\begin{tabular}{llll}
\hline & $\begin{array}{l}\text { Patients N=76 } \\
\text { Mean+SD }\end{array}$ & $\begin{array}{l}\text { Controls N=50 } \\
\text { Mean+SD }\end{array}$ & P-value \\
\hline $\operatorname{lgE}(\mathrm{IU} / \mathrm{ml})$ & $264.76 \pm 304.91$ & $39.15 \pm 27.22$ & $<0.001$ \\
M:L ratio & $0.49 \pm 0.21$ & $0.32 \pm 0.06$ & $<0.001$
\end{tabular}

P-value $<0.00$ I highly significant difference

Table 2 Distribution of the total immunoglobulin E (IgE) levels, according to age

\begin{tabular}{lll}
\hline Age group (years) & Mean \pm SD & P-value \\
\hline$<15$ & $392.56 \pm 486.14$ & \\
$15-29$ & $209.12 \pm 171.36$ & \\
$30-44$ & $161.25 \pm 91.25$ & \\
$\geq 45$ & $369.93 \pm+374.64$ & \\
\hline
\end{tabular}

P-value $<0.05$ statistically significant difference

The phagocytic activity of the monocytes at the time of monocytosis which reported as positive correlation with monocytes count during the infection (Figure 1). It might probably reflect the status of phagocytosis activity during the infection. The clear explanation is that M: $\mathrm{L}$ ratio form as an indicator of an individual's capacity ${ }^{12}$ to the rate of an effective immune response against malaria infection (Table 4).$^{10}$ That led to alteration of monocytes quantity, and a rising ratio of monocytes to the leukocyte count due to the increased phagocytic mechanism of monocytes. ${ }^{12,13}$ This make the ratio of monocytes to lymphocytes increase with the severity of the infection (Figure 1), we found it familiar with what previously mentioned and reported by Warim $\mathrm{GM}^{1}$ \& Döring $\mathrm{M}^{14}$ On the other hand, the total immunoglobulin $\mathrm{IgE}$ was estimated to check if there is a correlation on the second arm of immune response (humeral part). The total IgE particularly attributed to the unique activity necessary for ant parasitic defense. ${ }^{10}$ The result displayed that there were variation in the levels of total IgE, and it was highest than controls group in difference age categories (Figure 3) and this different was highly statistically significant. ${ }^{15,16}$ Total immunoglobulin IgE elevated to a high concentrations in young children and also the raising was reported in older patients (Table 2). IgE antibodies increased with age, becoming most significantly elevations in children less than 15years of age and in older more than 45-year age. In adult, those with Plasmodium falciparum malaria had significantly higher IgE antibody levels.

Table 3 Correlation of immunoglobulin E levels, TWBC and leukocyte differential with severity of malaria

\begin{tabular}{lll}
\hline Parameters & Correlation coefficient & P-value \\
\hline IgE & 0.49 & $0.000^{* *}$ \\
TWBC & -0.024 & 0.84 \\
Neutrophil \% & -0.101 & 0.39 \\
Lymphocyte \% & -0.055 & 0.64 \\
Monocyte\% & 0.237 & $0.039^{*}$ \\
Eosinophil\% & 0.209 & 0.070 \\
\hline
\end{tabular}

P-value $<0.05$ significant correlation, $* * P$-value $<0.00$ I strong correlation

Table 4 Association between ML ratio and total lgE level with severity of malaria

\begin{tabular}{lll}
\hline & HR $(95 \%$ Cl $)$ & P-value \\
\hline ML ratio & $0.30(0.11$ to 0.84$)$ & 0.022 \\
Total lgE & $0.99(0.997$ to 0.999$)$ & 0.009 \\
\hline
\end{tabular}

P-value $<0.05$ statistically significant association, $\mathrm{HR}=$ Hazard ratio

\section{Conclusion}

Ultimately malaria causing by Plasmodium falciparum parasites has a significant effect to immune system compounds. Admittedly we firmly found that the ratio of monocyte to lymphocyte (M: L) ratio, and total IgE which facilely obtained could contemplate the capability of effectiveness of immune response rate to P. falciparum infection. $\mathrm{M} / \mathrm{L}$ ratio it might help successfully in severity determination of malaria infection. $\operatorname{IgE}$ going to interact effectively to eliminate the pathogenicity of malaria infection.

\section{Availability of data and materials}

The datasets generated and/or analyzed during the current study are available from the corresponding author on reasonable request. 


\section{Funding}

No funding was received.

\section{Ethics approval and consent to participate}

This study was approved by the Research and Medical Ethics Committee Alzaim Al-Azhari University, Medical record office D, S H Hospital (No. 2017-07-T0021-R003).

\section{Acknowledgements}

This study was partly supported technically by Dr. Mohamed Mahjob Alfail (D, S, H) Lab Manager. We thank the Faculty of Medical Laboratory Sciences Alzaeim Al-azhari University, for their support. a special thanks to Professor. Maowia M. Mukhtar (institute of endemic disease) for guiding and supporting till this work brought to the light. We also thank the laboratory staff and nurses of Dar ALelaj Specialized Hospital for their help.

\section{Conflicts of interest}

The authors declare that they have no competing interests exist.

\section{References}

1. Warimwe GM, Murungi LM, Kamuyu G, et al. The ratio of monocytes to lymphocytes in peripheral blood correlates with increased susceptibility to clinical malaria in Kenyan children. PLoS One. 2013;8:e57320.

2. Schofield L, Grau GE. Immunological processes in malaria pathogenesis. Nature Reviews Immunology. 2005;5:722.

3. Kotepui M, Phunphuech B, Phiwklam N, et al. Effect of malarial infection on haematological parameters in population near ThailandMyanmar border. Malaria J. 2014;13:218.

4. Tangpukdee N, Yew HS, Krudsood S, et al. Dynamic changes in white blood cell counts in uncomplicated Plasmodium falciparum and P. vivax malaria. Parasitology International. 2008;57:490-494.

5. Tangpukdee N, Duangdee C, Wilairatana P, et al. Malaria diagnosis: a brief review. The Korean journal of Parasitology. 2009;47:93.

6. Eze EM, Christian SG. Immunoglobulin Levels in Plasmodium falciparum Malaria Infected Subjects in Port Harcourt, Nigeria. Int $J$ Adv Multidiscip Res. 2016;3:49-55.
7. Perlmann P, Perlmann H, Flyg BW, et al. Immunoglobulin E, a pathogenic factor in Plasmodium falciparum malaria. Infection and immunity. 1997;65:116-121.

8. Calissano C, Modiano D, Sirima BS, et al. IgE antibodies to Plasmodium falciparum and severity of malaria in children of one ethnic group living in Burkina Faso. The American journal of tropical medicine and hygiene. 2003;69:31-35.

9. Nag S, Bandyopadhyay S. White blood cell segmentation and malaria. International Journal of Current Medical and Pharmaceutical Research. 2018.

10. Antwi-Baffour S, Kyeremeh R, Buabeng D, et al. Correlation of malaria parasitaemia with peripheral blood monocyte to lymphocyte ratio as indicator of susceptibility to severe malaria in Ghanaian children. Malaria J. 2018;17:419.

11. Huh HJ, Oh GY, Huh JW, et al. Malaria detection with the Sysmex XE-2100 hematology analyzer using pseudoeosinophilia and abnormal WBC scattergram. Annals of hematology. 2008;87:755.

12. Khaing Swe K, Lei Lei Win, Thinn Yu Aung, et al. Approach to the Patients with Monocytosis. IOSR Journal of Dental and Medical Sciences. 2015;14(5):81-86.

13. Rogerson $\mathrm{S}$, Ortega-Pajares A. The rough guide to monocytes in malaria infection. Frontiers in immunology. 2018;9:2888.

14. Döring M, Cabanillas Stanchi KM, Erbacher A, et al. Phagocytic activity of monocytes, their subpopulations and granulocytes during posttransplant adverse events after hematopoietic stem cell transplantation. Immunobiology. 2015;220:605-613.

15. Seka-Seka J, Brouh Y, Yapo-Crézoit AC, et al. The role of serum immunoglobulin E in the pathogenesis of Plasmodium falciparum malaria in Ivorian children. Scandinavian journal of immunology. 2004;59:228-230.

16. Stanisic DI, Fowkes FJ, Koinari M, et al. Acquisition of antibodies against Plasmodium falciparum merozoites and malaria immunity in young children and the influence of age, force of infection, and magnitude of response. Infection and immunity. 2015;83:646-660. 